Témoigner Témoigner. Entre histoire et mémoire

Getuigen Revue pluridisciplinaire de la Fondation Auschwitz

$122 \mid 2016$

Révisionisme et négationisme

\title{
Représentations mémorielles au Pérou : de la défense associative des droits de l'homme à la convocation des arts
}

Memorial representations in Peru: the voluntary defence of human rights for the convening of the arts

\section{Mylène Herry}

\section{(2) OpenEdition \\ Journals}

\section{Édition électronique}

URL : https://journals.openedition.org/temoigner/4863

DOI : $10.4000 /$ temoigner.4863

ISSN : 2506-6390

Traduction(s) :

Memorial representations in Peru: The voluntary defence of human rights for the convening of the arts - URL : https://journals.openedition.org/temoigner/4920 [en]

Éditeur :

Éditions du Centre d'études et de documentation Mémoire d'Auschwitz, Éditions Kimé

\section{Édition imprimée}

Date de publication : 2 mai 2016

Pagination : 185-191

ISSN : 2031-4183

\section{Référence électronique}

Mylène Herry, «Représentations mémorielles au Pérou : de la défense associative des droits de I'homme à la convocation des arts », Témoigner. Entre histoire et mémoire [En ligne], 122 | 2016, mis en ligne le 30 septembre 2021, consulté le 02 février 2022. URL : http://journals.openedition.org/ temoigner/4863; DOI : https://doi.org/10.4000/temoigner.4863 


\section{$\checkmark$ Site mémoriel}

\section{REPRÉSENTATIONS \\ MEMORIELLES AU PÉROU: \\ DE LA DÉFENSE ASSOCIATIVE \\ DES DROITS DE L'HOMME A \\ LA CONVOCATION DES ARTS}

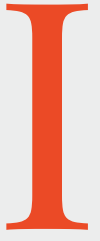

1 s'agit dans cet espace consacré aux sites mémoriels d'élargir la problématique des représentations de la mémoire dans le Pérou actuel, introduite dans le numéro précédent à travers la présentation de El ojo que llora, monument emblématique érigé à Lima en 2005. En effet, rappelons que le Pérou est un pays qui a subi pendant deux décennies (19802000) un conflit interne d'une extrême violence. Il est nécessaire de souligner la longue période de latence existante entre le présent de la guerre, la vérité quant aux faits reconnus et le moment de la transmission testimoniale engagée, entre autres, par la CVR dès 2001. C'est alors que jaillit la Vérité historique, celle, portée par les témoins directs des exactions, des témoins incarnés par les paysans andins, les sans voix, le plus souvent victimes de la violence politique et raciale. L'histoire officielle jusque-là relayée par le pouvoir étatique est revisitée et corrigée dans l'espoir de construire une mémoire collective. Des représentations mémorielles, aussibien artistiques, associatives, architecturales que culturelles, naissent dans une perspective de réparation nationale. Cependant, les convictions, les peurs, les rancœurs ou les regrets des uns et des autres face aux choix faits et/ou (non) assumés pendant le conflit armé tendent à marquer des divergences profondes. Dans ce sens, El ojo que llora, nous l'avons vu, està la fois un exemple d'appel à la communion nationale à partir d'un projet commémoratif et un exemple de rejet de cette communion cristallisé par les tensions nationales autour des responsabilités et la légitimité des autorités et celles du Parti communiste Sentier Lumineux.

Je propose dans cette même perspective de faire un état géné- ral, sans pour autant le considérer exhaustif, des représentations mémorielles dans le Pérou postconflit en considérant tous les champs informatifs engagés. Il s'agira avant tout de présenter l'Association Nationale des Familles des Séquestrés, Détenus et Disparus du Pérou (ANFASEP), qui fut créée en 1983 à Ayacucho, ville et région andines les plus durement touchées pendant le conflit armé.Il sera opportun ici de citer quelques lieux mémoriels très emblématiques de cette période et de cette zone géographique, lieux que l'on retrouve fréquemment représentés dans la littérature testimoniale. Aussi, nous citerons le travail de l'association citoyenne Para que no se repita qui œuvre pour combattre l'oubli et les injustices subies pendant et depuis cette période noire de l'histoire péruvienne. Enfin, nous ferons état des espaces culturels consacrés au conflit interne. 
Local de l'association ANFASEP à Ayacucho Rez-de-chaussée : ancien comedor de los niños Salle d'exposition d'objets appartenant aux victimes (Musée de la mémoire - Ayacucho) Dessin sur un mur intérieur du local.

\section{AYACUCHO - ANFASEP}

L'Association Nationale des Familles des Séquestrés, Détenus et Disparus du Pérou (ANFASEP) est créée le 2 septembre 1983 à Ayacucho, tout d'abord sous le nom de Comité des Familles de Disparus (CFD). Cette association est un prolongement de l'Association APRODEH (Asociación PRo DErechos Humanos) basée à Lima et engagée dans le respect et la défense des Droits de l'Homme au Pérou. Alors que le climat d'insécurité est à son paroxysme au début du conflit, «Mamá Angélica », alias Angélica Mendoza de Ascarza, décide de créer cette association suite aux nombreuses plaintes de disparitions non élucidées, entre autres, celle de son fils. Cette association, avant-gardiste face à la montée de la violence, réunit 350 membres autour de son combat pour localiser les séquestrés et retrouver les corps des victimes. Les témoignages soulignent des abus d'autorité et une négation des Droits de l'Homme. Ce lieu devient un lieu de rassemblement, de confession et de partage pour tous ceux qui, de près ou de loin, ont subi les traumatismes de la guerre interne.

En 1985, et pour répondre au nombre croissant d'orphelins de guerre, une cantine appelée « Comedor de niños

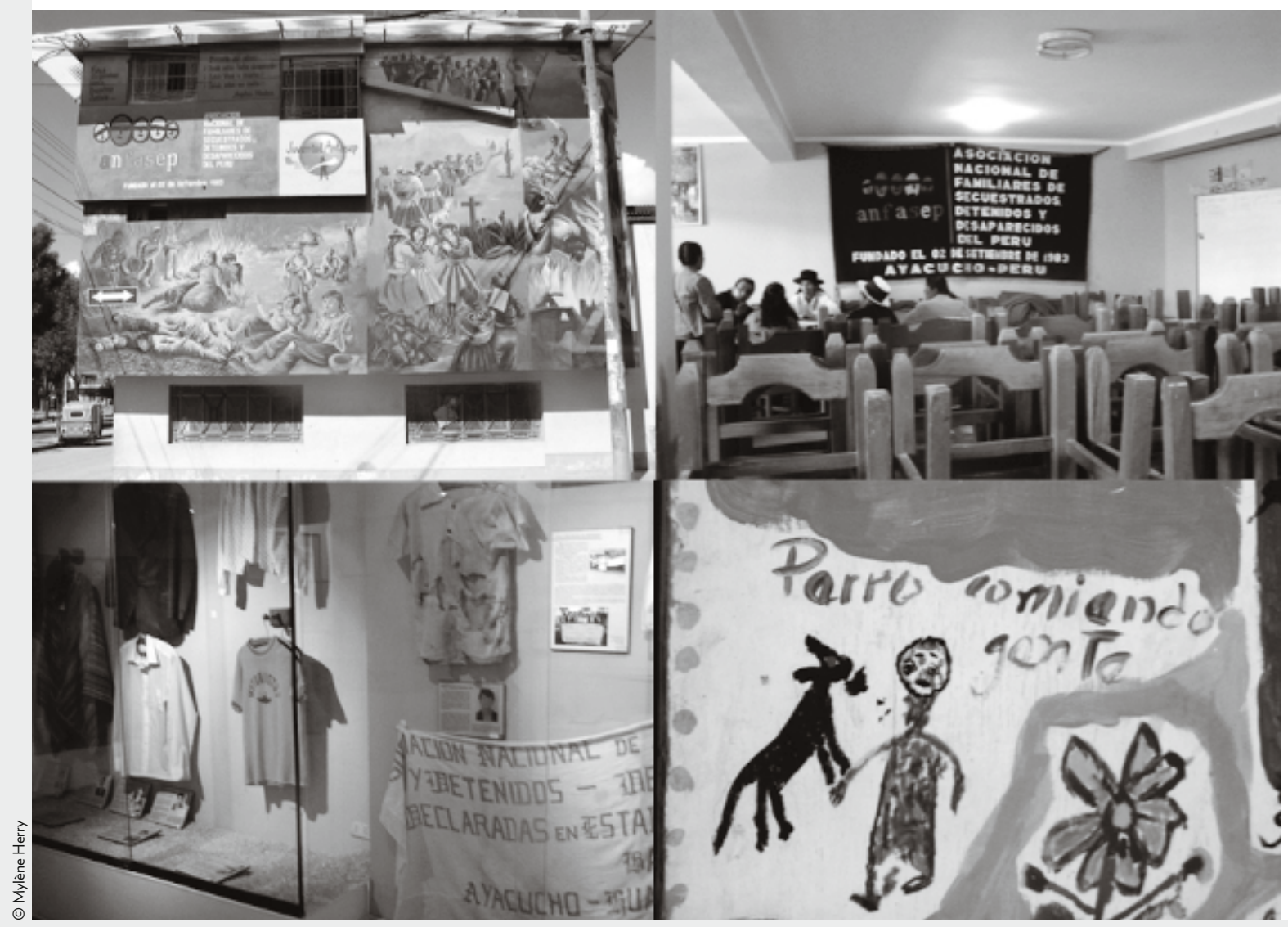


Adolfo Pérez Esquivel» - en hommage à son parrain, prix Nobel de la paix s'ouvre dans ces mêmes murs. Elle fermera en 1996 à la suite d'un déficit budgétaire grandissant.

Deux ans après la publication du Rapportfinal delaCVR, en 2005, et grâce aux appuis institutionnels et associatifs, le musée de la mémoire est créé au troisième étage du local. Il s'organise en trois espaces : la zone du «chaos » avec des témoignages des années de terreur et une reconstitution historique de la période ; une zone d'exposition de photos, d'objets et d'affaires personnels des disparus; une dernière zone quiprésente les photos des membres de l'association, essentiellement des femmes seules à la suite de la disparition de leur mari et/ ou de leurs enfants. Des fresques de neuf mètres sur six recouvrent les murs extérieurs du bâtiment. Ces peintures représentent des scènes de la violence politique subie. Elles furent dessinées par Claudio Martinez, artiste de l'école des Beaux-Arts Felipe Guamán Poma de Ayala d'Ayacucho. Nous pouvons aussi apprécier les photos des disparus sur la façade, les noms de victimes inscrits sur quelques pierres à l'entrée du musée, le sanctuaire d'ANFASEP, ou bien encore le parc et le totem de la mémoire (érigé également en 2005) sur la place voisine. Ces manifestations mémorielles témoignent du passé violent de la région et de la volonté de la population d'une reconnaissance de celui-ci. Par exemple, le monument en fer que représente le totem de la mémoire, expose trois côtés symbolisant le passé violent d'une population prise entre deux feux, un présent judiciaire dont la balance ne s'équilibre toujours pas et le futur autour de la figure de la colombe et des deux mains qui s’apprêtent à se réconcilier.

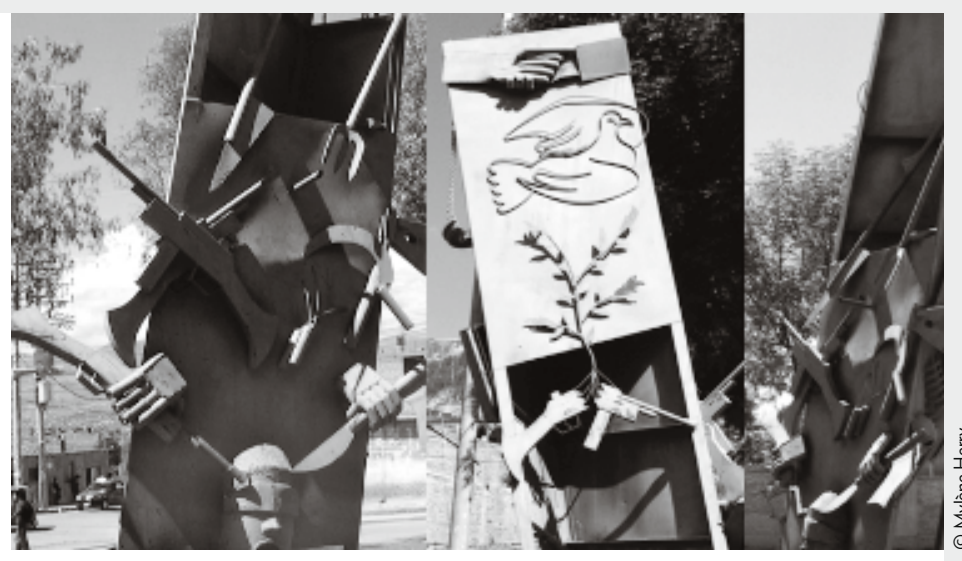

Aussi, l'ouverture en 2006 de la boutique artisanale dans le musée et la création de nombreux retablos relatifs à cette période permettent une diffusion majeure du message de l'association et, par conséquent, une activation de la mémoire collective.

\section{AYACUCHO}

À Huamanga - Ayacucho, les nombreux vestiges des années 1980 rappellent, d'une part, la forte idéologie marxiste aux alentours de l'ancienne faculté et, d'autre part, la violence du conflit (restes de bâtiments incendiés ou soufflés). Ce lieu est symbolique si l'on considère le rôle qu'ont joué les universités tout au long du conflit. Dès la fin des années 1970, le Sentier Lumineux recrute dans les universités et les instituts éducatifs, et prépare ainsi l'imposition de son idéologie dans la décennie suivante. La population estudiantine est très active et opère souvent en faveur de la révolution populaire. En représailles, et jusqu'à la fin du gouvernement de Fujimori, les interventions militaires se multiplient. Elles sont symboliques dans l'effacement, par exemple, de l'image du Che sur la façade de la faculté de droit de San Marcos (Lima); elles sont barbares quand le groupe parami- • • 


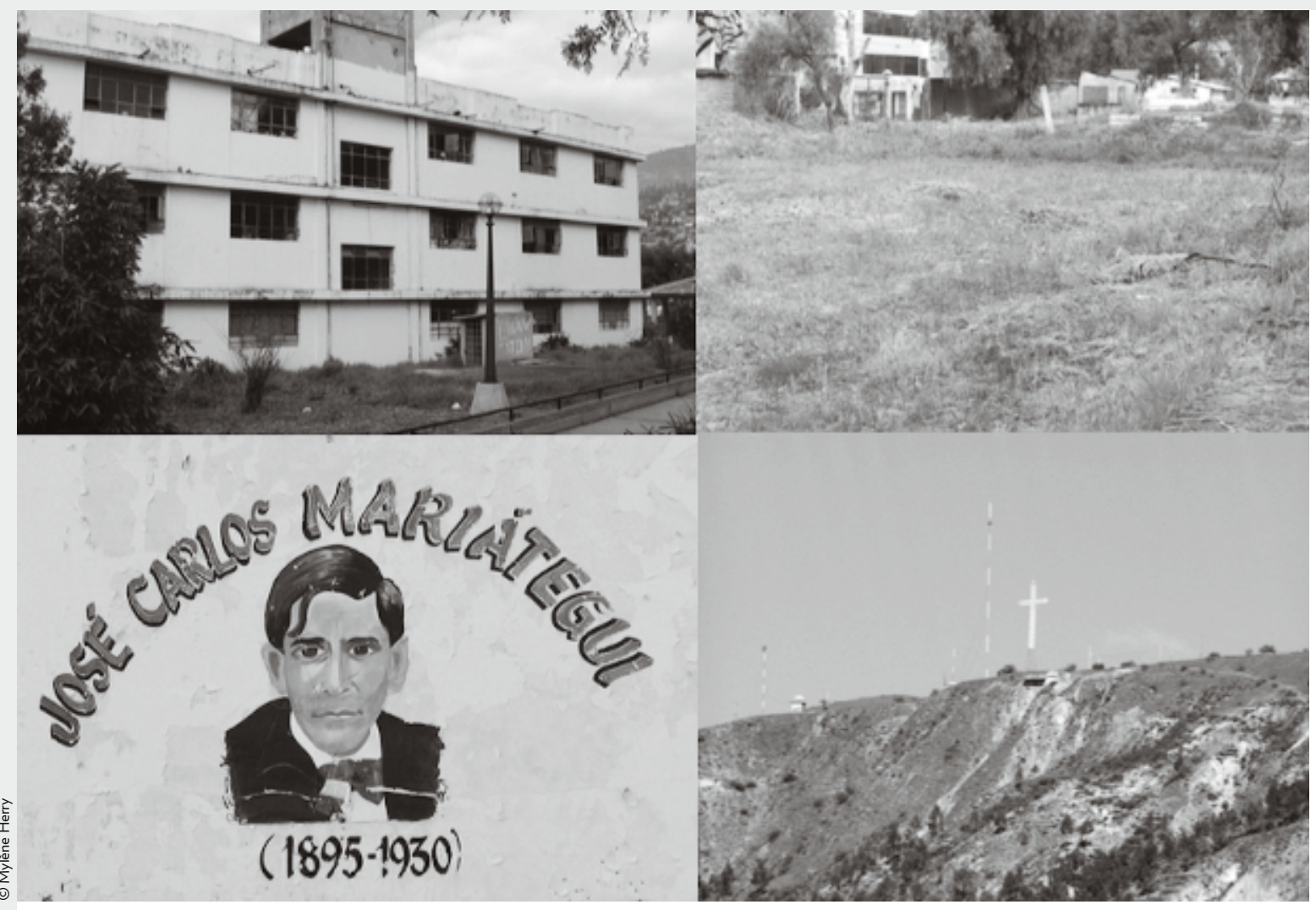

$\uparrow$ Vestiges de l'ancienne Université de Huamanga dont les bâtiments portent encore les noms des leaders politiques d'obédience communiste La Cruz de La Paz.
•• litaire, appelé «Colina», séquestre le 18 juillet 1992 neuf étudiants et un professeur de l’Université Enrique Guzmán y Valle, La Cantuta. Leurs corps seront retrouvés un an plus tard dans une fosse commune à Cieneguilla (Lima).

De même, la «Cruz de la Paz», symbole de la ville, fut construite en 2011, à l'initiative de la municipalité, sur el Cerro de la Picota. Elle s'édifie avec les restes des tours électriques détruites lors du conflit. Elle domine la ville et s'illumine tous les soirs à 18 heures, rappelant les feux du Parti communiste péruvien - Sentier Lumineux -, qui, depuis ces collines, imposait le couvrefeu, en plongeant la ville dans l'obscurité. Plus récemment, en juillet 2011, «la
Cruz de la Hoyada » fut inaugurée sur le site de l'ex-caserne de Los Cabitos à Ayacucho, en mémoire des victimes. Cette appellation fait référence aux fosses communes retrouvées à cet endroit ainsi qu'aux pratiques de crémation des corps dans les fours prévus à cet effet.

Depuis la diffusion du rapport de la CVR, il faut souligner la propension en littérature à réinvestir ces lieux qui deviennent le théâtre d'un roman policier (Abril rojo, 2007), l'inspiration poétique de voix-témoins (Rocio Silva Santisteban, Las hijas del terror, 2005) ou encore le modèle pour une représentation artistique et graphique des faits (Jesús, Cossío, Rupay, 2008 - Barbarie, 2010). La littérature devient une littéra- ••• 

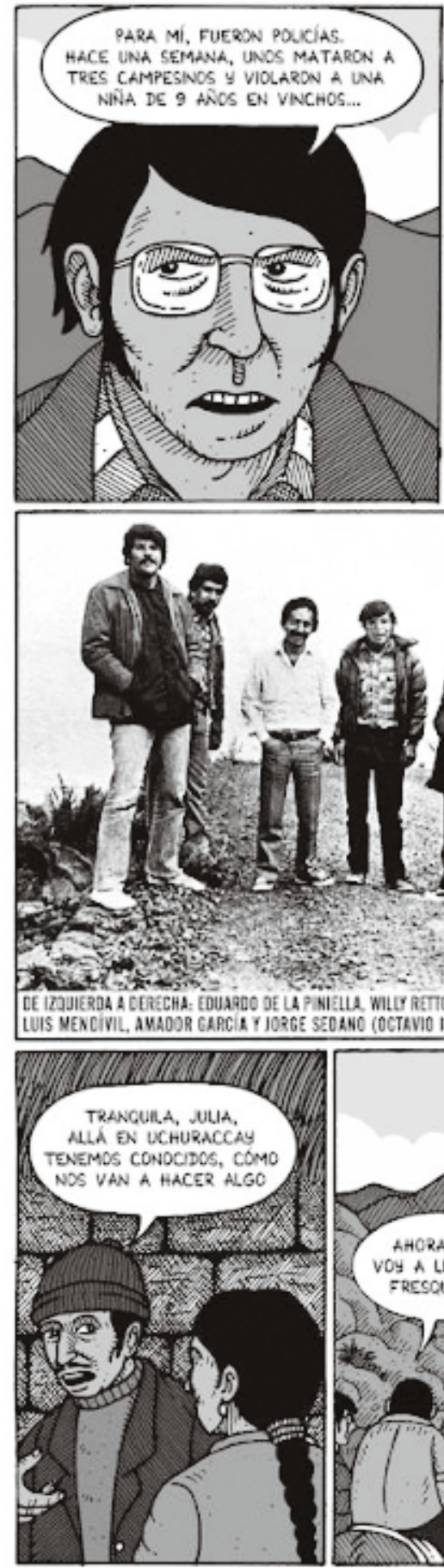
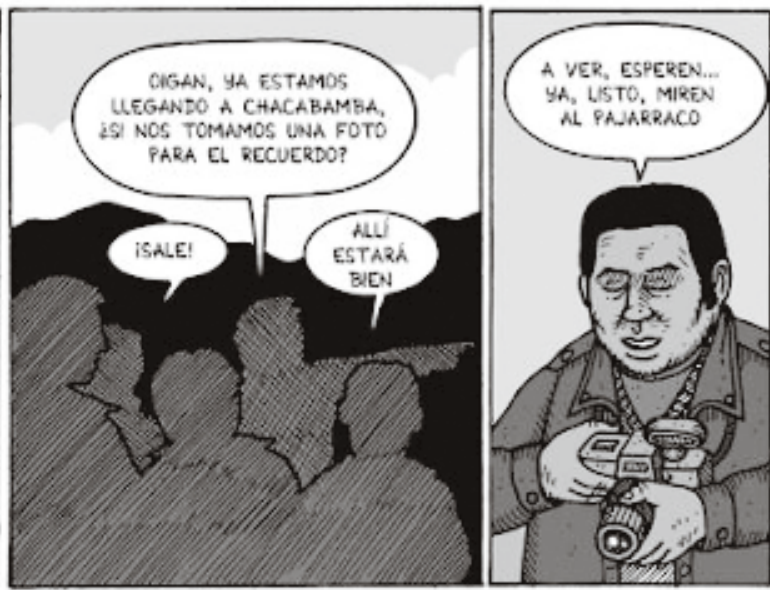

EN CHRCABAMBa, INFRNTE CONVECE A SI HEDIO HERMENO ILAN ARCUMEDO FRRA CUE LOS GUIE HASTA LCRURACCAY. LA FAMILU DE AFGL HEDO LES DR DE COUER Y LES PRESTA NANTAS Y UNA MULA PAPA CLECLR CANLRAS Y BUITCS.
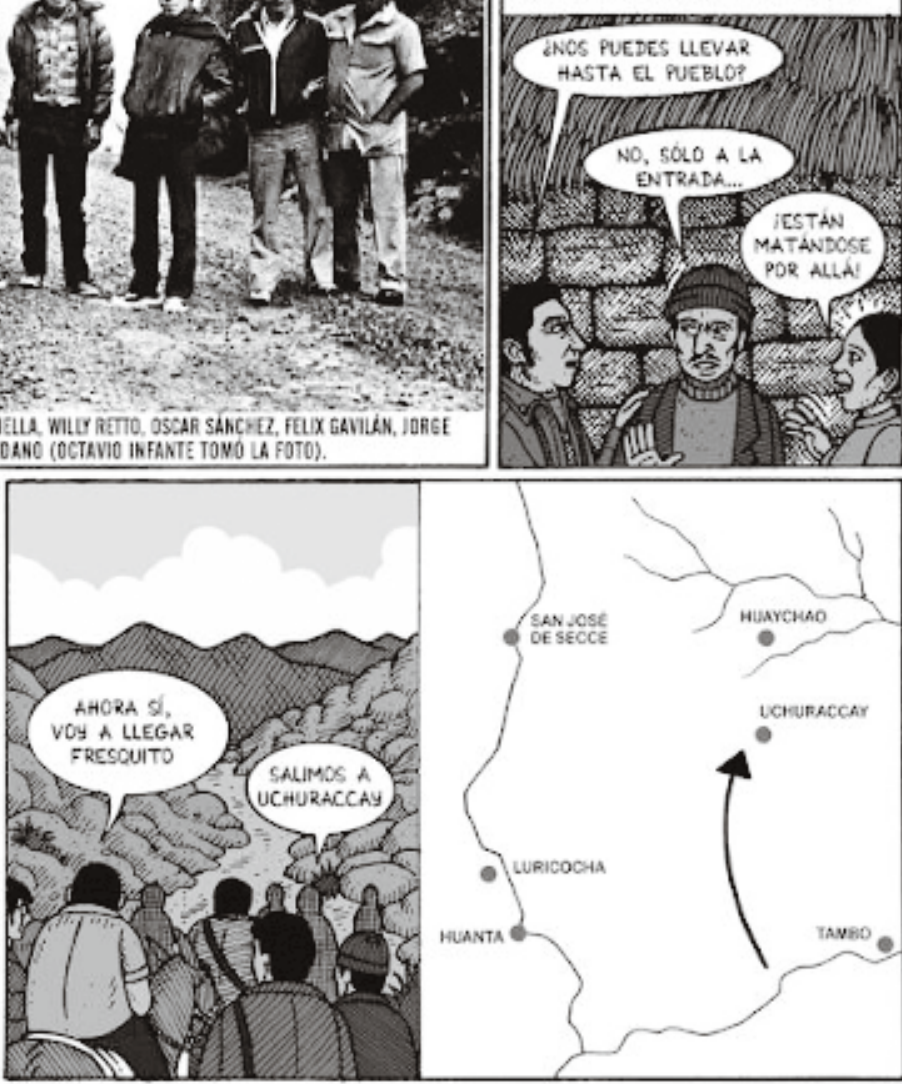

$\rightarrow$ Planche de Rupay qui inclut, à titre journalistique, informatif et testimonial, la photo des huit journalistes tués à Uchuraccay. 
$\bullet \bullet$ ture qui prête sa voix aux témoins dans le texte comme dans sa structure qui suggère une hybridité des genres et une interdiscursivité testimoniale. Rupay propose un support photographique récurrent tout au long de l'œuvre, support qui tend à prouver les faits et, ainsi, conforter la thèse de la CVR.

\section{PARA QUE NO SE REPITA}

L'association citoyenne Para que no se repita («Pour que cela ne se répète pas») réalise depuis 2004, année de sa création, un suivi du développement et de la mise en application des recommandations de la CVR. Elle mobilise une coalition de plus de 400 organisations des Droits de l'Homme et groupes communautaires dans les 25 régions du pays qui travaillent sur le terrain. Elle s'efforce de répondre actuellement aux demandes d'assistance pour organiser des événements de commémorations et l'édification de sites pour victimes et survivants. De fait, les sujets centraux soutenus par des campagnes nationales tournent autour de l'éducation, des Droits de l'Homme, de la jeunesse, des moyens de communication... Il s'agit de tout mettre en place pour réussir une authentique Réconciliation nationale dans une cohabitation sans violence, ni exclusion.

\section{LA CONVOCATION DE L'IMAGE}

L'exposition Yuyanapaq, au sixième étage du musée de la Nation à Lima, propose l'histoire du conflit interne péruvien à travers 182 photos réalisées par la CVR lors de ses investigations. La publication consécutive du récit en images de ces années de terreur semble être un moyen de ne pas oublier. On compte de plus en plus de manifestations artistiques relatives à cette période noire. Par exemple, Santiago Quintanilla inaugure en 2008 une galerie d'art graphique - Lo mejor está por venir («Le meilleur est à venir») -, dont les œuvres hybrides (fiction/réalité) sont créées à l'aide de journaux nationaux et de bandes dessinées, et organisées sous forme chronologique.

Depuis 2009, Elmuseo virtual de arte y memoria (MVAM) est une mine d'informations sur les nouvelles publications, expositions et les nouveaux films (et vidéos) ou projets autour de l'art et de la mémoire. Karen Bernedo Morales, la directrice de cet espace virtuel, nous propose non seulement une actualité

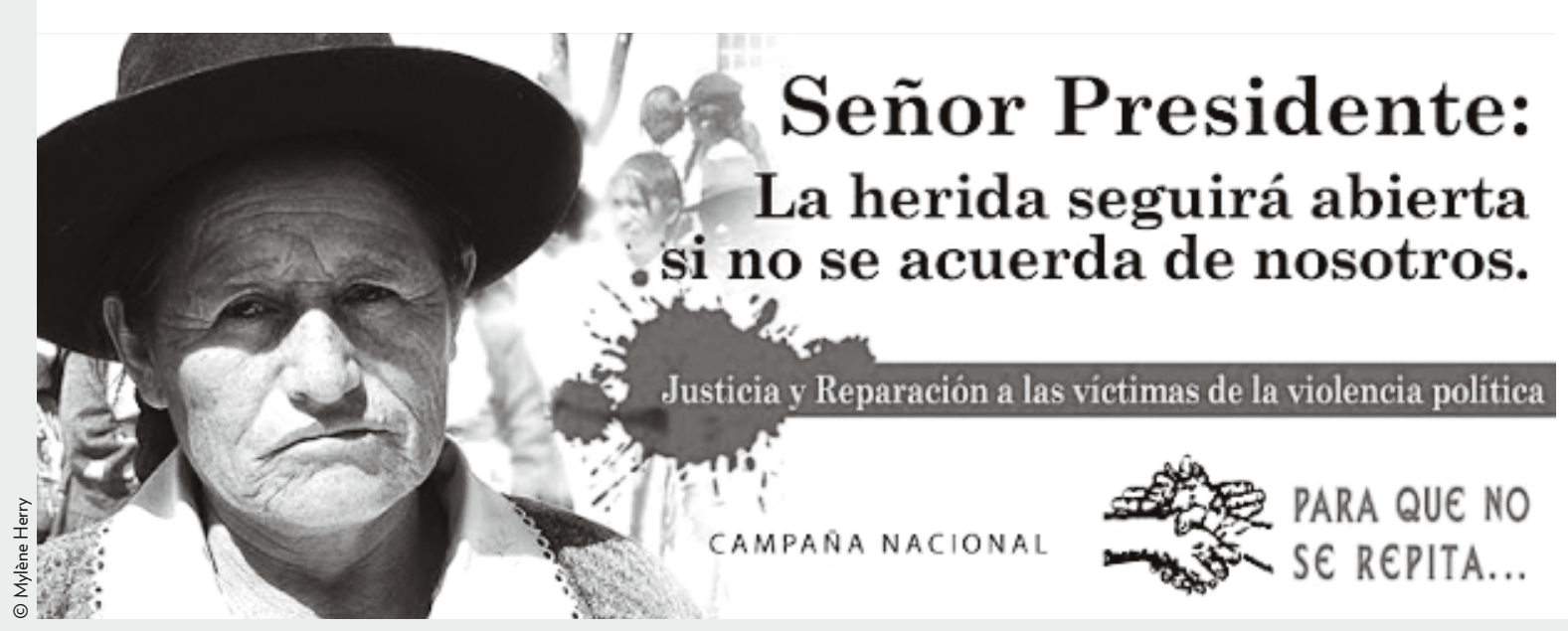


culturelle nationale et internationale autour des manifestations artistiques de la mémoire, mais aussi un espace de recherches autour des problématiques mémorielles à travers des articles et une bibliographie.

À Lima - Miraflores, El Lugar de la Memoria del Perú sera un musée en hommage aux victimes du conflit armé au Pérou (1980-2000), financé en grande partie par le gouvernement allemand qui à la suite de sa visite en 2008 fit une donation de deux millions d'euros pour soutenir la construction et la maintenance d'un musée de la mémoire. La Comisión de Alto Nivel, chargée de l'organisation, est, depuis octobre 2010, présidée par le peintre péruvien Fernando de Szyszlo, successeur de Mario Vargas Llosa. Un site a vu le jour récemment sous le nom de Lugar de la Memoria, la Tolerancia y la Inclusión Social (LUM) sur un terrain de sept hectares le long de la côte depuis le quartier de San Martín jusqu’à Miraflores. Aujourd'hui, la direction, incarnée par Denise Ledgard, discute avec le comité d'organisation (Víctor Vich, Ponciano del Pino, Jorge Villacorta et Natalia Iguiñiz) des expositions du processus participatif dont les grands axes sont développés dans un livre intitulé Los fundamentos conceptuales del LUM, publié en 2015. Son inauguration est prévue avant la fin de l'année 2015.

Ces quelques exemples de manifestations sont une forme de témoignagehommage à toutes les victimes et leurs familles de ces années de terreur. Ces créations permettent de pérenniser l'information et de construire une mémoire collective autour d'un passé longtemps caché - et encore parfois nié - pour une éventuelle réconciliation. I

Mylène Herry

\section{Bibliographie}

Le site de l'Association Para que no se repita :

http://www.paraquenoserepita.org.pe/

consulté régulièrement depuis 2011 (dernière connexion : septembre 2015).

Le site de l'Association ANFASEP:

http://www.anfasep.org/anfasep/index.php?cmbldioma=es,

consulté régulièrement depuis 2011 (dernière connexion : septembre 2015).

Le site du musée virtuel en ligne (mvam):

http://www.museoarteporlasmemorias.pe/category/tags/per\%C3\%BA consulté le 7 octobre 2015.

Hatun, Willakuy, [versión abreviada del informe final de la Comisión de la Verdad y Re-conciliación ed.], Lima, 2014.

Edilberto Jiménez, Quispe (2005) : Chungui : violencia y trazos de memoria, Lima, COMISEDH.

José, Ponciano Del Pino ; Carlos Agüero (2014) : Cada uno, un lugar de memoria. Fundamentos Conceptuales del Lugar de la Memoria,

la Tolerancia y la Inclusion Social, Lima, LUM, consulté en ligne le 7 octobre 2015 :

http://lugardelamemoria.org/wp-content/uploads/2015/01/LIBRO-LUM.pdf

ANFASEP (éd.) (2007) : ¿Hasta cuándo tu silencio? testimonios de dolor y coraje, Lima.

APRODEH (2001) : El reto de la verdad y la justicia. Perú, 1980-2000, Lima, Federación Internacional de Derechos Humanos.

APRODEH (2002) : Verdad, memoria, justicia y reconciliación : sociedady comisiones de la verdad, Lima, Asociación pro derechos humanos, Aprodeh.

COMISIÓN DE LA VERDAD Y RECONCILIACIÓN (2003):

Yuyanapaq : para recordar. Relato visual del conflicto armado interno en el Perú (1980-2000), Lima, PUCP.

$\checkmark$ «Yuyanapaq : para recordar 1980-2000. Relato visual del conflicto armado interno en el Perú », Boletín Educación Sin Fronteras, Lima, n 18, 1995, consulté en ligne en 2010 et téléchargé en format PDF :

www.educacionsinfronteras.org

\footnotetext{
$\rightarrow$ Références littéraires citées

Cossío, Jesús (2010) : Barbarie, Lima, Contracultura.

Roncagliolo, Santiago (2007): Abril rojo, Madrid, Alfaguara.

Rossell, Luis ; Villar, Alfredo ; Cossío, Jesús (2009): Rupay, Lima,

La oveja roja.

Silva Santisteban, Rocío (2007) : Las hijas del terror, Lima, Copé.
} 